\title{
Outcomes of Tracheostomy in COVID-19 Patients: A Single Centre Experience
}

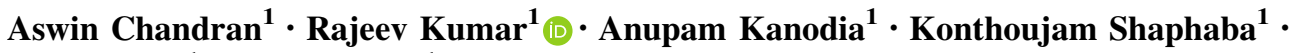 \\ Prem Sagar $^{1} \cdot$ Alok Thakar $^{1}$
}

Received: 27 April 2021 / Accepted: 4 July 2021/Published online: 13 July 2021

(C) Association of Otolaryngologists of India 2021

\begin{abstract}
The rapid emergence of COVID-19 has led to increase in the number of patients requiring prolonged ventilation and tracheostomy. Though global guidelines are evolving, there is a paucity of literature about optimal timing and outcome after tracheostomy in COVID-19 patients. We set out to assess the clinical characteristics and outcome of COVID-19 patients who underwent tracheostomy.This single-center, prospective observational cohort study analyzed all the consecutive tracheostomy performed on COVID-19 patients from April 12020 to January 31,2021 . The primary outcome measure was the 30-day mortality rate following tracheostomy and association with various prognostic risk factors. The secondary outcome measures included various tracheostomy-related events, perioperative complications, and decannulation rate.The study included 51 patients with a median age of 52 years and the majority were male (62.74\%). 62.74\% (32 of 51) tracheostomies were done early (within 10 days of intubation) and the mean duration from endotracheal intubation to tracheostomy was 10.27 days. The 30-day mortality rate was $66.66 \%$ (34 of 51) with no tracheostomy-related mortality and the mean duration between tracheostomy and death was 8.29 days. The presence of sepsis was associated with a higher rate of mortality $(p=0.002)$ while the timing of tracheostomy was not related to increased mortality $(p=0.365)$. The most common perioperative complication was tracheostoma bleeding (16.64\%). At a median follow-up of 7.5 months,
\end{abstract}

Rajeev Kumar

rajeev9843@yahoo.co.in

1 Department of Otorhinolaryngology and Head and Neck Surgery, All India Institute of Medical Sciences, New Delhi 110029, India only four patients were decannulated.Though associated with a higher rate of 30-day mortality rate, tracheostomy among COVID-19 patients is a safe and an effective weaning tool.

Keywords Covid-19 · Tracheostomy · Outcomes · Timing of tracheostomy $\cdot$ Mechanical ventilation

\section{Introduction}

The novel coronavirus disease 2019 (COVID-19), has rapidly emerged into a catastrophic global pandemic causing unprecedented healthcare challenges. The prior clinical reports had shown that approximately 5-15\% of patients with COVID-19 require intubation and mechanical ventilation during hospitalization [1-3]. The surge in the number of patients requiring prolonged ventilator support necessitated an increase in the number of tracheostomies performed, which is considered beneficial for early weaning from the ventilator. Tracheostomy helps in decreasing the need for sedation, improvement in work of breathing and pulmonary toileting, reducing ventilator-associated pneumonia and airway stenosis allows early mobilization, reduces the ICU stay, and improves the patient comfort. In the context of the ongoing pandemic, tracheostomy aids in the transition of a patient from ICU level care to early rehabilitation especially in resource constraint nations with limited ICU spaces and manpower, and also aids in reducing the generation of highly infectious aerosols associated with high-flow oxygen or other modes of noninvasive ventilation [4-7]. The potential for generation of contagious aerosols during the procedure and post-tracheostomy care causing an occupational health hazard to health care workers and overall poor prognosis of patients 
requiring ICU care makes tracheostomy among COVID-19 patients a futile intervention [3, 8-11].

Albeit several guidelines and recommendations addressing the safety of tracheostomy in COVID-19 patients have been published in the literature, the usefulness of tracheostomy is still a topic of debate in terms of indications, timing, techniques, and outcome [12, 13]. The timing of tracheostomy among COVID-19 patients represents the most debatable topic as available literature yet to reach a consensus. The proven benefits of early tracheostomy among non-covid patients including low mortality rate, lower incidence of ventilator pneumonia or airway stenosis, and shorter ICU stay have not yet been established among COVID-19 patients requiring tracheostomy. Given the aforestated benefits and risks to performing tracheostomies in COVID-19 patients, we intend to describe our experience at a single, tertiary care center performing tracheostomies in patients with COVID19. In this prospective study, we aim to describe the clinicdemographic characteristics, complications, morbidity, mortality, and outcomes of COVID-19 patients who underwent tracheostomy. We also analyzed the factors predicting mortality in this group of patients.

\section{Materials and Methods}

Study design and patient selection: A single-institution, prospective, observational cohort study was conducted on COVID-19 intubated patients in intensive care units (ICU) who underwent tracheostomy at a COVID-19 designated tertiary care center between April 1, 2020, and January 31, 2021. All patients had a documented diagnosis of COVID19 confirmed using nasal polymerase chain reaction (PCR) swab test and underwent open surgical tracheostomy by an otorhinolaryngologist. All the tracheostomies were performed by open surgical methods by the otolaryngologists in a dedicated COVID-19 well ventilated operating room which works under negative pressure. We followed standard protocols for limiting the generation of infectious aerosols and level III PPE was worn by the entire operating team while performing tracheostomy The study was approved by the institutional review board and informed written consent was waived.

Data collection: The electronic medical records of patients were reviewed for demographic and clinical data of the patients who underwent open surgical tracheostomy during the study period. Socio-demographic and clinical data included age, sex, and medical comorbidities of patients. Medical comorbidities reviewed included hypertension, coronary artery disease, diabetes mellitus, cerebrovascular accident, chronic kidney disease, chronic pulmonary disease, malignancy, neurological disease, and presence of sepsis during a hospital stay. We also collected tracheostomy-related parameters like timing of tracheostomy, perioperative and postoperative complication, duration of the procedure, and mechanical ventilation history. The timing of tracheostomy was classified as early (within 10 days of intubation) and late (more than 10 days of intubation). The tracheostomy-related complications were categorized as intraoperative, immediate (within $24 \mathrm{~h}$ ) and late post-operative. The primary outcome measure was 30-day mortality rate following tracheostomy and association with potential prognostic risk factors like age, gender, presence of neurological disease, hypertension, and diabetes mellitus, a combination of 2 or more comorbidities, presence of sepsis, the timing of tracheostomy (early vs Late), and timing of tracheostomy after COVID-19 positivity. The secondary outcome measures were the length of ICU stay, days from confirmed COVID-19 to tracheostomy, days from intubation to tracheostomy, days from tracheostomy to ICU and hospital discharge, perioperative complications, risk of infection to the operating team, and decannulation rate.

Data analysis: Data were expressed as count (percentage, \%), median, and mean (standard deviation). Comparisons were performed using Fischer's exact test for categorical variables and a $p$-value of less than 0.05 was considered significant.

\section{Results}

From April 12020 to January 31, 2021, 51 mechanically ventilated COVID-19 positive patients underwent tracheostomy at our institute. The study population ranged from 23 to 83 years of age (median: 52 years). $62.74 \%$ (32 of 51) patients were male and $37.36 \%$ (19 of 51) were female. The duration of follow-up from the date of tracheostomy ranged from 15 days to 12 months with a median follow-up period of 7.5 months. The most common co-existing medical illness was neurological disease $(50.98 \%, 26$ of 51$)$ followed by hypertension $(33.33 \%, 17$ of 51) and diabetes mellitus (29.41\%, 15 of 51). The clinicdemographic characteristics of the study population were summarized in Table 1.

37 patients $(72.55 \%)$ tracheostomies were undertaken within 14 days of COVID-19 positivity. Thirty-two $(62.74 \%)$ patient's tracheostomies were performed early i.e. within 10 days of intubation. The average time between the intubation date and the tracheostomy date was 10.27 days (range, 5-20 days). 24 patients $(47.05 \%)$ patients were liberated from mechanical ventilation following tracheostomy and the average number of days from tracheostomy to ventilator weaning was 7.5 days (range, 1-18 days). The average number of ICU stay pre- 
Table 1 Patient's clinic-demographic characteristics

\begin{tabular}{ll}
\hline Patient Characteristics $(n=51)$ & Number $(\%)$ \\
\hline Age (median; range) & $52 ; 23-83$ years \\
Gender (Male; Female) & $32(62.74) ; 19$ \\
& $(37.26)$ \\
Comorbidities & \\
Neurological disease & $26(50.98)$ \\
Hypertension & $17(33.33)$ \\
Diabetes mellitus & $15(29.41)$ \\
Renal disease & $5(9.80)$ \\
Pulmonary disease & $4(7.8)$ \\
Cardiovascular disease & $4(7.8)$ \\
Malignancy & $4(7.8)$ \\
Indication for tracheostomy & \\
$\quad$ Prolonged ventilation & $47(92.15)$ \\
$\quad$ Upper airway obstruction & $2(3.92)$ \\
Pulmonary toileting & $2(3.92)$ \\
Presence of sepsis & $25(49.01)$ \\
Early tracheostomy (within 10 days of intubation) & $32(62.74)$ \\
Number of tracheostomies within 14 days of & $37(72.55)$ \\
COVID-19 positivity & \\
\hline
\end{tabular}

tracheostomy was 11.5 days (range, $4-23$ days) while posttracheostomy was 8.5 days (range, $1-20$ days). The timing of tracheostomy events was summarized in Table 2 .

The 30-day mortality rate was $66.66 \%$ (34 of 51) with no tracheostomy-related mortality. Of these, $23(67.64 \%)$ tracheostomies were performed early and $11(32.36 \%)$ late. The mean duration between tracheostomy and death was 8.29 days (range, 1-21 days). Seven patients expired in ICU after weaning from the ventilator whereas the rest of the patients succumbed to illness while on mechanical ventilation. The presence of sepsis in COVID-19 patients was associated with a higher rate of mortality ( $p$-value $=$ 0.002 ) in our cohort. The age of the patient (more than 40 years) and presence of neurological diseases were also associated with a higher rate of mortality in our study,

Table 2 The timing of tracheostomy events

\begin{tabular}{ll}
\hline Tracheostomy events & Mean (range in days) \\
\hline Hospital admission to tracheostomy & $12.56(7-24)$ \\
Intubation to tracheostomy & $10.27(5-20)$ \\
ICU admission to tracheostomy & $11.5(4-23)$ \\
Tracheostomy to weaning from the ventilator & $7.5(1-18)$ \\
Tracheostomy to ICU discharge & $8.5(1-20)$ \\
Tracheostomy to death (Non-survivors) & $8.29(1-21)$ \\
Tracheostomy to discharge (Survivors) & $9.52(7-29)$ \\
\hline
\end{tabular}

though statistically not significant (p-value, 0.076 and 0.074 respectively). Compared with tracheostomies conducted after 10 days of intubation, tracheostomies within 10 days were associated with an increased mortality rate, though statistically not significant $(p$ value $=0.365$ ). The association between various prognostic factors and mortality after tracheostomy is summarized in Table 3.

The most common intraoperative complication was bleeding (more than $100 \mathrm{ml}$ ), which occurred in 6 $(11.76 \%)$ patients and controlled using bipolar cauterization or vessel ligation. The immediate postoperative complications were peristomal bleeding $(5.88 \%, 3$ of 51$)$, tracheostomy-tube block $(3.92 \%, 2$ of 51$)$, tracheostoma infection $(3.92 \%, 2$ of 51), and pneumothorax (1.96\%, 1 of 51). No late postoperative complications were noted till the end of the study follow-up period. Among the survivors, all patients were weaned off from the ventilator (mean duration: 7.11 days, range: $2-15$ days), shifted out from ICU (mean duration: 8.5 days, range 3-16 days), and discharged from hospital (mean duration: 13 days, range: 3-31 days). At the time of submission, four patients were decannulated (4 of 17, 23.52\%), and the mean duration between tracheostomy and decannulation was 3.5 months. The COVID-19 tracheostomy complications and outcomes are shown in Table 4.

The mean number of days from tracheostomy to ICU discharge among survivors was significantly higher $(13.00 \pm 7.36)$ than the mean number of days from tracheostomy to death among non-survivors $(8.29 \pm 5.31)$ with a p-value of 0.011 . However, the number of days from admission to tracheostomy, ICU admission to tracheostomy, intubation to tracheostomy, and tracheostomy to weaning from ventilator carries no significance between survivors and non-survivors (Table 5).. The average time of the first change of the tracheostomy tube was ranged from 10 to 21 days (average 13.84 days). Among the survivors, only 4 patients were successfully decannulated on subsequent follow-up visits with a mean period for a tracheostomy to decannulation was 3.5 months. To date, none of our tracheostomy team has developed any symptoms suggestive of COVID-19 nor tested positive for COVID19.

\section{Discussion}

Our study represents the largest series of tracheostomies undertaken among COVID-19 patients in India so far and it elaborates our experience performing tracheostomies on 51 patients during the peak of the pandemic in our nation. Extensive lung injury caused by COVID-19 which demands prolonged ventilation, multiple failed extubation trial during the course of illness due to airway edema, 
Table 3 The association between prognostic risk factors and mortality after tracheostomy

\begin{tabular}{|c|c|c|c|c|}
\hline \multirow[t]{2}{*}{ Prognostic variable } & \multirow[t]{2}{*}{ Category } & \multicolumn{2}{|c|}{ Number of patients } & \multirow[t]{2}{*}{$p$-value } \\
\hline & & Non-survivors & Survivors & \\
\hline \multirow[t]{2}{*}{ Age } & More than 40 years & 29 & 10 & 0.076 \\
\hline & Less than 40 years & 5 & 7 & \\
\hline \multirow[t]{2}{*}{ Gender } & Male & 20 & 12 & 0.542 \\
\hline & Female & 14 & 5 & \\
\hline \multirow[t]{2}{*}{ Diabetes mellitus } & Yes & 11 & 4 & 0.745 \\
\hline & No & 23 & 13 & \\
\hline \multirow[t]{2}{*}{ Hypertension } & Yes & 10 & 7 & 0.530 \\
\hline & No & 24 & 10 & \\
\hline \multirow[t]{2}{*}{ Neurological disease } & Yes & 14 & 12 & 0.074 \\
\hline & No & 20 & 5 & \\
\hline \multirow[t]{2}{*}{ Comorbid illness } & More than two & 16 & 7 & 0.770 \\
\hline & Less than or equal to two & 18 & 10 & \\
\hline \multirow[t]{2}{*}{ Presence of Sepsis } & Yes & 22 & 3 & 0.002 \\
\hline & No & 12 & 14 & \\
\hline \multirow[t]{2}{*}{ Day of tracheostomy after intubation } & Early (within 10 days) & 23 & 9 & 0.365 \\
\hline & Late ( after 10 days) & 11 & 8 & \\
\hline \multirow[t]{2}{*}{ Days of tracheostomy after COVID-19 diagnosis } & Within 14 days & 27 & 10 & 0.183 \\
\hline & After 14 days & 7 & 7 & \\
\hline
\end{tabular}

Bold represents various prognostic factors and their correlation with mortality of the patient

muscle weakness, and reduced cough reflex indicates far greater benefits of tracheostomy among these cohorts than the traditionally ventilated patients. However, tracheostomy among COVID-19 patients is challenging considering the severity of the infection and associated hypoxemia, technical difficulties while performing the procedure donning personal protective equipment (PPE), and hazard of transmission of infection to health care workers [14]. In the context of the limited number of guidelines for safe tracheostomy among COVID-19 patients based on real experience, we do believe our study will contribute to the paucity of existing literature.

Table 4 COVID-19 tracheostomy complications and postoperative outcome

\begin{tabular}{ll}
\hline Complications & Number $(\%)$ \\
\hline Intraoperative bleeding $(>100 \mathrm{ml})$ & $6(11.76)$ \\
Post-operative peristomal bleeding & $3(5.88)$ \\
Tube block & $2(3.92)$ \\
Tracheostoma infection & $2(3.92)$ \\
Pneumothorax & $1(1.96)$ \\
Outcomes & \\
Expired & $34(66.66)$ \\
Survived and discharged & $17(33.34)$ \\
Decannulated & $4(7.84)$ \\
\hline
\end{tabular}

Since the patients with critical illness due to COVID-19 induced respiratory failure carry a dismal prognosis the decision to perform tracheostomy must be based on defined prognostic indicators $[10,13]$. . Tracheostomy among COVID-19 patients has the advantage of early ventilator weaning, decrease the ICU stay, and free up available ICU resources [14]. However, Shiba et al. had suggested that due to the rapid evolution of disease which leads to poor

Table 5 Comparison of tracheostomy-related events between survivors and non-survivors

\begin{tabular}{llll}
\hline Tracheostomy events & $\begin{array}{l}\text { Mean } \pm \text { SD } \\
\text { duration in Non- } \\
\text { survivors }\end{array}$ & $\begin{array}{l}\text { Mean } \pm \text { SD } \\
\text { duration in } \\
\text { Survivors }\end{array}$ & $\begin{array}{l}P \text { - } \\
\text { value }\end{array}$ \\
\hline $\begin{array}{l}\text { Admission to } \\
\text { tracheostomy }\end{array}$ & $12.29 \pm 2.95$ & $13.12 \pm 2.09$ & 0.309 \\
$\begin{array}{l}\text { Intubation to } \\
\text { tracheostomy }\end{array}$ & $10.09 \pm 2.54$ & $10.65 \pm 2.89$ & 0.482 \\
$\begin{array}{l}\text { ICU admission to } \\
\text { tracheostomy }\end{array}$ & $10.94 \pm 3.12$ & $10.35 \pm 2.96$ & 0.389 \\
$\begin{array}{c}\text { Tracheostomy to } \\
\text { weaning from the } \\
\text { ventilator }\end{array}$ & $7.74 \pm 4.45$ & $7.12 \pm 3.72$ & 0.429 \\
$\begin{array}{l}\text { Tracheostomy to ICU } \\
\text { discharge/death }\end{array}$ & $8.29 \pm 5.31$ & $13.00 \pm 7.36$ & $\mathbf{0 . 0 1 1}$ \\
\hline
\end{tabular}

Bold represents trachestomy related parameters among survivors and non-survivors 
prognosis among patients with severe COVID-19 requiring ICU stay, tracheostomy doesn't confer any benefit to the outcome [15]. In our series, the major indications for tracheostomy were prolonged ventilation $(92.15 \%)$ similar to other published studies, and the decision to proceed with a tracheostomy was based on the assessment of prognosis and risk-benefit analysis on case to case basis by the opinion of the ICU physicians and otolaryngologists [13]. The mean age of COVID-19 patients who underwent tracheostomy in this study was 51.39 years, and the majority were male $(62.74 \%)$. This high number of males who underwent tracheostomy and succumbed to illness posttracheostomy ( 20 of $34,58.82 \%$ ) could be due to the poor prognosis described for the male population in recent publications [1-3].

The timing of tracheostomy among COVID-19 patients is another intriguing topic and several guidelines on this topic yet to reach a consensus. Earlier recommendations suggested delaying tracheostomy beyond 14 days when the risk of viral transmission is less and the prognosis of the critically ill patient becomes clearer [9, 12, 16, 17]. Studies on SARS-Cov-2 viral load during the course of illness had suggested that individuals are more contagious in the earlier stage of disease corresponding with high viral load in upper respiratory tract secretions and had predicted reduced rate of infection beyond 10-days of symptoms onset [18]. Additionally, performing an early tracheostomy would make prone invasive ventilation difficult in the early course of diseases and severely hypoxemic patients wouldn't tolerate the loss of positive airway pressure during the procedure $[13,19]$. Though deferring the tracheostomy of COVID-19 patients decreases the hazard of acquiring infection among the health care workers, prolonged tracheal intubation and mechanical ventilation might cause other complications like airway stenosis, ventilator-associated pneumonia, longer ICU stay, etc. Early tracheostomy also increases the availability of ICU beds as tracheostomised patients can be cared for in nonICU wards and decrease the duration of use of the sedative drug among such patients [17, 20]. Though COVID-19 patients requiring mechanical ventilation carries a poor prognosis, tracheostomy may be indicated early in lifesaving situations or situation in which tracheostomy might improve the survival of patient $[8,10]$. A Chinese multicenter study had suggested that compared with tracheostomies conducted after 14 days of intubation, tracheostomies within 14 days were associated with an increased mortality rate [21]. However, a prospective cohort study among 50 patients had reported early tracheostomy ( $<10$ days) bears no association with mortality as highlighted in our study [22]. Since data on prognostic factors of chronically ventilated COVID-19 patients are lacking, much of the recent recommendations are an extrapolation of benefits and survival data on non-COVID patients requiring chronic ventilation. However, these studies had suggested contrasting results on benefits of early tracheostomy in terms of reduced mortality rate, shorter duration of ICU stay than late tracheostomy [4, 23, 24]. A recent systematic review and meta-analysis on non-COVID 19 patients showed that early tracheostomy (within 7 days) was associated with a reduction in mortality rate and ventilator associated pneumonia, duration of mechanical ventilation, and duration of ICU stay [25, 26]. In contrast, a previous randomized clinical trial of nonCOVID-19 patients did not reveal an improved mortality rate or reduced length of ICU stay on mechanically ventilated patients who underwent early tracheostomy [24].

Prior studies had shown that otolaryngologists, anesthesiologists, and nurses caring for patients with tracheostomy are at heightened risk of acquiring COVID-19 infection with an odds ratio of 4.2 [27]. The data on viral load risk would suggest delaying the tracheostomy procedure beyond 14-21 days after the onset of symptoms when the patient's viral load becomes negligible and would represent the safest balance between the survival of patients and risk for viral transmission [9, 28]. The low contagious nature of late tracheostomy (beyond 14 days) was further ascertained by Kai Xy et al. who showed no detectable SARS-CoV-2 RNA in the surgical environment in patients who underwent delayed tracheostomy [29]. However, there is a lack of direct supporting evidence regarding the safety of performing late tracheostomy with regard to nosocomial transmission and further studies are needed to understand attendant risks of infectious exposure to health care workers. In our series, the mean period of intubation before tracheostomy was 10.27 days and 32 $(62.74 \%)$ patient's tracheostomies were performed early (within 10 days of intubation) without waiting for 2-3 weeks as other studies suggest. The relatively early tracheostomy in our study could be due to the limited availability of ICU beds to accommodate the increased patient load during the height of the pandemic and to fasten the period of weaning from the ventilator. It confers additional benefits of a shorter period of mechanical ventilation and ICU stay compared to delayed tracheostomy group. In our opinion, the decision for tracheostomy among critically ill COVID-19 patients must be made with an individualizing approach after a multidisciplinary evaluation of the benefits or risks of tracheostomy. We suggest that earlier tracheostomy should be considered for patients who were unable to be weaned by a ventilator but carries a good chance for overall survival ideally around 10 days of intubation which correlates with the anticipated decrease in viral transmission. Although tracheostomy confers benefit for carefully selected COVID-19 mechanically ventilated patients, we defer the procedure in patients who require 
high ventilator requirements with high fractions of inspired oxygen $(>80 \%)$, significant medical comorbidities leading to poor survival, severe ARDS with a low chance of recovery. The mortality in patients with COVID-19 undergoing tracheostomy had been reported to vary from 7 to $41 \%$ in the previously published outcome studies $[16,30]$. The overall 30-day mortality in our study was $66.66 \%$, mirroring the results of other studies that reported $>50 \%$ mortality rate for COVID-19 patients who required prolonged ventilation culminating in a tracheostomy $[8,10]$. The higher rate of mortality in our study indicates the higher prevalence of comorbid illness in our cohort which has been shown to increase the COVID-19 associated complications. $35.29 \%$ (18 of 51) of our study population expired within 7 days of the tracheostomy, a far higher mortality rate seen in the first week following tracheostomy under normal conditions. The presence of sepsis, older age, and coexisting neurological diseases were associated with poor outcomes in our series similar to previous studies [10]. Though the timing of tracheostomy (early vs late) in our study is not associated with a higher mortality rate, the alarming rate of overall and 7-days post tracheostomy mortality among critically ill patients suggests delaying the tracheostomy beyond 10-days of intubation in patients with poor prognostic factors. We reported no major immediate or delayed complications of tracheostomy, and more importantly, no deaths were related to the procedure. The incidence of tracheostomy complications in our series was $15.68 \%$ (8 of 51) and our low rate complications emphasize the importance of standardized technique and a dedicated operating team including surgeon and anesthetists. Perioperative bleeding probably due to administration of anti-coagulation therapy owing to prothrombotic state among COVID-19 patients, mucous plugging of tracheostomy tube due to more copious and viscid secretion, and peristomal skin ulceration were the commonest complication noted in this series. Though tracheostomy increases the risk of nosocomial transmission among healthcare workers as they are exposed to high levels of contagious aerosols none of our health care personals involved in tracheostomy were infected with COVID-19. The nosocomial transmission risk can be successfully mitigated by appropriate PPE and adequate surgical techniques by highly experienced surgical teams adhering to infection control recommendations. A relatively small sample size which precludes performing detailed data analysis, the probable under-reporting of outcomes as the data was retrieved from electronic medical records are the few limitations of this study.

\section{Key messages}

Though associated with a higher rate of mortality, the timing, and selection of a patient for tracheostomy can be made flexible based on the health resources to accommodate the increased patient in ICU as in our series. We suggest an experienced multi-disciplinary tracheostomy team is of paramount importance to perform a safe tracheostomy in COVID-19 patients and to limit the risk of occupational transmission and improve the overall prognosis. Future studies are warranted to identify the ideal timing and patient prognostic risk factors associated with better survival outcomes in COVID-19 patients undergoing a tracheotomy.

Authors' Contributions All authors contributed to the study conception and design. Material preparation, data collection and analysis were performed by AC, AK and KS. The first draft of the manuscript was written by AC and critical revision was done by RK, PS. AT has done the final modifications the manuscript to be submitted. All authors read and approved the final manuscript.

Source of Funding The author(s) received no financial support for the research, authorship, and/or publication of this article.

\section{Declarations}

Conflict of interest The author(s) declared no potential conflicts of interest with respect to the research, authorship, and/or publication of this article.

Ethical Approval All procedures performed in studies involving human participants were in accordance with the ethical standards of the institutional and/or national research committee and with the 1964 Helsinki declaration and its later amendments or comparable ethical standards.

Consent to Participate Informed written consent was waived off by institute ethics committee.

\section{References}

1. Guan W, Ni Z, Hu Y et al (2020) Clinical characteristics of coronavirus disease 2019 in China. N Engl J Med 382:1708-1720

2. Huang C, Wang Y, Li X et al (2020) Clinical features of patients infected with 2019 novel coronavirus in Wuhan. China Lancet 395(10223):497-506

3. Richardson S, Hirsch JS, Narasimhan M et al (2020) Presenting characteristics, comorbidities, and outcomes among 5700 patients hospitalized with COVID-19 in the New York City area. JAMA 323(20):2052-2059

4. Hosokawa K, Nishimura M, Egi M, Vincent JL (2015) Timing of tracheotomy in ICU patients: a systematic review of randomized controlled trials. Crit Care 19:424

5. Andriolo BN, Andriolo RB, Saconato H, Atallah AN, Valente O (2015) Early versus late tracheostomy for critically ill patients. Cochrane Database Syst Rev 1:CD007271.

6. Siempos II, Ntaidou TK, Filippidis FT, Choi AMK (2015) Effect of early versus late or no tracheostomy on mortality and 
pneumonia of critically ill patients receiving mechanical ventilation: a systematic review and meta-analysis. Lancet Respir Med 3:150-158

7. Tran K, Cimon K, Severn M, Pessoa-Silva CL, Conly J (2012) Aerosol generating procedures and risk of transmission of acute respiratory infections to healthcare workers: a systematic review. PLoS One 7:e35797

8. Yang X, Yu Y, Xu J et al (2020) Clinical course and outcomes of critically ill patients with SARS-CoV-2 pneumonia in Wuhan, China: a single-centered, retrospective, observational study. Lancet Respir Med 8:475-481

9. Givi B, Schiff BA, Chinn SB et al (2020) Safety recommendations for evaluation and surgery of the head and neck during the COVID-19 pandemic. JAMA Otolaryngol-Head Neck Surg 146:579-584

10. Zhou F, Yu T, Du R et al (2020) Clinical course and risk factors for mortality of adult inpatients with COVID-19 in Wuhan, China: a retrospective cohort study. Lancet 395:1054-1062

11. Adams JG, Walls RM (2020) Supporting the health care workforce during the Covid-19 global epidemic. JAMA 323:1439-1440

12. Takhar A, Walker A, Tricklebank S et al (2020) Recommendation of a practical guideline for safe tracheostomy during the COVID-19 pandemic. Eur Arch Otorhinolaryngol 277(8):2173-2184

13. Mcgrath BA, Brenner MJ, Warrillow SJ et al (2020) Tracheostomy in the COVID-19 era: global and multidisciplinary guidance. Lancet Respir Med 8:717-725

14. Mattioli F, Fermi M, Ghirelli M et al (2020) Tracheostomy in the COVID-19 pandemic. Eur Arch Otorhinolaryngol 277(7): 2133-2135

15. Shiba T, Ghazizadeh S, Chhetri D, St John M, Long J (2020) Tracheostomy considerations during the COVID-19 pandemic. OTO Open 4(2):2473974X20922528. Published 2020 Apr 21.

16. Chao TN, Braslow BM, Martin ND et al (2020) Tracheotomy in ventilated patients with COVID-19. Ann Surg 272:30-32

17. David AP, Russell MD, El-Sayed IH, Russell MS (2020) Tracheostomy guidelines developed at a large academic medical center during the COVID-19 pandemic. Head Neck 42:1291-1296

18. Wölfel R, Corman VM, Guggemos W et al (2020) Virological assessment of hospitalized patients with COVID-2019. Nature 581:465-469

19. Volo T, Stritoni P, Battel I et al (2021) Elective tracheostomy during COVID-19 outbreak: to whom, when, how? Early experience from Venice. Italy Eur Arch Otorhinolaryngol 278(3):781-789

20. Zhang X, Huang Q, Niu X et al (2020) Safe and effective management of tracheostomy in COVID-19 patients. Head Neck 42(7):1374-1381

21. Tang Y, Wu Y, Zhu F et al (2020) Tracheostomy in 80 COVID19 Patients: a multicenter, retrospective, observational study. Front Med (Lausanne) 7:615845

22. Avilés-Jurado FX, Prieto-Alhambra D, González-Sánchez $\mathrm{N}$ et al (2020) Timing, complications, and safety of tracheotomy in critically Ill patients with COVID-19 JAMA Otolaryngol Head Neck Surg 147(1):1-8.

23. Schultz MJ, Pattnaik R, Dondorp AM (2020) Walking the line between benefit and harm from tracheostomy in COVID-19. Lancet Respir Med 8:656-657

24. Young D, Harrison DA, Cuthbertson BH et al (2013) Effect of early vs late tracheostomy placement on survival in patients receiving mechanical ventilation: the TracMan randomized trial. JAMA 309(20):2121-2129

25. Adly A, Youssef TA, El-Begermy MM, Younis HM (2018) Timing of tracheostomy in patients with prolonged endotracheal intubation: a systematic review. Eur Arch Otorhinolaryngol 275:679-690

26. Wang R, Pan C, Wang X, Xu F, Jiang S, Li M (2019) The impact of tracheotomy timing in critically ill patients undergoing mechanical ventilation: a meta-analysis of randomized controlled clinical trials with trial sequential analysis. Heart Lung 48(1):46-54

27. Cheung JC, Ho LT, Cheng JV, Cham EYK, Lam KN (2020) Staff safety during emergency airway management for COVID-19 in Hong Kong. Lancet Respir Med 8(4):e19

28. Sommer DD, Engels PT, Weitzel EK et al (2020) Recommendations from the CSO-HNS taskforce on performance of tracheotomy during the COVID-19 pandemic. J Otolaryngol Head Neck Surg 49(1):23

29. Xu K, Zhang XH, Long XB, Lu X, Liu Z (2021) An environmental study of tracheostomy on eight COVID-19 patients. J Otolaryngol Head Neck Surg 50(1):3

30. Thal AG, Schiff BA, Ahmed Y et al (2021) Tracheotomy in a high-volume center during the COVID-19 pandemic: evaluating the surgeon's risk. Otolaryngol Head Neck Surg 164(3):522-527

Publisher's Note Springer Nature remains neutral with regard to jurisdictional claims in published maps and institutional affiliations. 